\section{Aberrant $R$ heb-mediated mTORC1 activation and Pten haploinsufficiency are cooperative oncogenic events}

\author{
Caterina Nardella, ${ }^{1,2}$ Zhenbang Chen, ${ }^{1,2}$ \\ Leonardo Salmena, ${ }^{1,2,6}$ Arkaitz Carracedo, ${ }^{1,2,6}$ \\ Andrea Alimonti, ${ }^{1,2}$ Ainara Egia, ${ }^{1,2}$ \\ Brett Carver, ${ }^{2,3}$ William Gerald, ${ }^{4}$ \\ Carlos Cordon-Cardo, ${ }^{2,5}$ and \\ Pier Paolo Pandolfi ${ }^{1,2,7}$
}

${ }^{1}$ Cancer Genetics Program, Beth Israel Deaconess Cancer Center, Departments of Medicine and Pathology, Beth Israel Deaconess Medical Center, Harvard Medical School, Boston, Massachusetts 02115, USA; ${ }^{2}$ Cancer Biology and Genetics Program, Sloan-Kettering Institute, New York, New York 10021, USA; ${ }^{3}$ Department of Surgery, Division of Urology, Memorial Sloan-Kettering Cancer Center, New York, New York 10021, USA; ${ }^{4}$ Department of Pathology, Memorial Sloan-Kettering Cancer Center, New York, New York 10021, USA; ${ }^{5}$ Department of Pathology, Columbia University, New York, New York 10032, USA

The mammalian target of rapamycin (mTOR) represents a critical signaling crossroad where pathways commonly disrupted in cancer converge. We report here that Rheb GTPase, the upstream activator of the mTOR complex 1 (mTORC1) is amplified in human prostate cancers. We demonstrate that $R$ heb overexpression promotes hyperplasia and a low-grade neoplastic phenotype in the mouse prostate while eliciting a concomitant senescence response and a negative feedback loop limiting Akt activation. Importantly, we show that Pten haploinsufficiency cooperates with Rheb overexpression to markedly promote prostate tumorigenesis. We conclude that $\mathrm{Rheb}$ acts as a proto-oncogene in the appropriate genetic milieu and signaling context.

Supplemental material is available at http://www.genesdev.org.

Received March 3, 2008; revised version accepted June 10, 2008.

Signaling pathways that activate the mammalian target of rapamycin (mTOR) are frequently deregulated in prostate cancer $(\mathrm{CaP})$, in addition to various other cancers (Majumder and Sellers 2005; Easton and Houghton 2006). Activation of mTOR is a central component downstream from the phosphatidylinositol-3-kinase (PI3K)/AKT signaling cascade, a pathway that is counteracted by the tumor suppressor phosphatase and tensin

[Keywords: Rheb; Pten haploinsufficiency; prostate tumorigenesis; negative feedback loop; senescence]

${ }^{6}$ These authors contributed equally to this work.

${ }^{7}$ Corresponding author.

E-MAIL ppandolf@bidmc.harvard.edu; FAX (617) 667-0610.

Article is online at http://www.genesdev.org/cgi/doi/10.1101/gad.1699608. homolog deleted on chromosome 10 (PTEN). The PI3K/ AKT pathway is aberrantly activated in many $\mathrm{CaP}$ due to loss of function of at least one PTEN allele, an event reported to occur in $\sim 30 \%-70 \%$ of primary CaP (Gray et al. 1998; Whang et al. 1998). Furthermore, from studies of a "hypomorphic Pten allelic series" in mice we revealed that the prostatic epithelium is exquisitely vulnerable to subtle activation of Akt/mTOR signaling, which promotes prostate cancer initiation and progression (Di Cristofano et al. 2001; Trotman et al. 2003).

mTOR is an essential serine/threonine kinase that regulates protein synthesis, cell growth, and proliferation in response to pleiotropic inputs including growth factors, nutrients, energy, and stress (Wullschleger et al. 2006). mTOR differentially regulates PI3K/AKT signaling by acting as a key component of two multiprotein complexes: mTOR complex 1 (mTORC1), which is activated downstream from AKT, and mTORC2, which has been demonstrated to phosphorylate AKT (Guertin and Sabatini 2007). Moreover, in many cell types, mTOR has been reported to elicit a negative feedback regulation on the PI3K pathway through the ability of its downstream target ribosomal S6 kinase 1 (S6K1) to inhibit IRS-1 (for review, see Guertin and Sabatini 2007). The elusive cross-talk between the AKT and mTOR pathways was uncovered by the finding that tuberous sclerosis complex 1 (TSC1) and TSC2 negatively regulate mTORC1 (Tapon et al. 2001; Gao et al. 2002). These studies demonstrated that AKT phosphorylates and inactivates the TSC1/TSC2 complex and, as a consequence, results in mTORC1 activation (Manning et al. 2002; Potter et al. 2003; Tee et al. 2003). Specifically, Ras homolog-enriched in brain (Rheb), a small guanosine triphosphate (GTP)-binding protein, was discovered as a novel substrate for TSC2, which could also lead to the activation of mTOR (Garami et al. 2003; Inoki et al. 2003; Zhang et al. 2003). TSC2 was shown to have a GTPase-activating protein (GAP) activity toward the Rheb GTPase; this event stimulates the intrinsic GTP hydrolysis activity of Rheb to promote its transition from an active GTP bound to an inactive guanosine diphosphate (GDP)bound form (Garami et al. 2003; Inoki et al. 2003; Zhang et al. 2003). Conversely, inactivation of the TSC1/TSC2 complex by AKT phosphorylation results in GTP loading and activation of Rheb, which ultimately promotes the activation of mTORC1 (for reviews, see Manning and Cantley 2003; Li et al. 2004). AKT also promotes mTORC1 activity through phosphorylation of PRAS40, which prevents its inhibitory function on mTORC1 (for review, see Guertin and Sabatini 2007). A recent report has shown that Rheb activates mTORC1 by opposing its endogenous inhibitor, FKBP38 (Bai et al. 2007). As a consequence, mTORC1 promotes cell growth through phosphorylation of various regulators of translation including the well-characterized ribosomal S6K1, which activates the S6 ribosomal protein (S6), and the eukaryotic translation initiation factor 4E-binding protein 1 (4EBP1), which leads to its uncoupling from the elongation initiation factor 4E (eIF4E) (for review, see Wullschleger et al. 2006).

Given the role of $\mathrm{mTORC1}$ activation in cancer initiation and progression, and the function of Rheb in promoting this activity, overexpression of $R$ heb could in principle have severe tumorigenic consequences. How- 
ever, evidence in support of this hypothesis is thus far lacking. Here we report that Rheb can indeed act as a proto-oncogenic GTPase and cooperate with PTEN haploinsufficiency in prostate tumorigenesis.

\section{Results and Discussion}

To investigate the contribution of Rheb in human CaP, we analyzed the occurrence and the frequency of genetic events involving $R$ heb in human $\mathrm{CaP}$ specimens $(n=186)$ by comparative genomic hybridization (CGH) for genetic alterations involving the region of $R$ heb on chromosome 7 . CGH analysis revealed that chromosome 7 is gained in $7.5 \%$ (14 of 186) of human prostate cancer specimens (Fig. 1A; W. Gerald, unpubl.). Of these 14 cases, six also demonstrated amplification of the region of Rheb on chromosome 7 with greater than single copy number gain (Fig. 1A).

In addition, we analyzed the level of Rheb protein in a panel of human $\mathrm{CaP}$ cell lines. Western blot analysis showed that protein levels of Rheb are increased in six of seven $\mathrm{CaP}$ cell lines compared with two nontumorigenic immortalized prostate epithelial lines (RWPE-1 and PWR-1E) used as a control (Fig. 1B). We quantified Rheb mRNA in the cancer cell lines with the highest expression at protein level and we observed that only two of six show increased transcription of Rheb (Fig. 1C). Taken together, these findings suggest that $R$ heb overexpression can occur at both a genomic and post-transcriptional level. Based on these observations, we hypothesized that Rheb overexpression is associated with human $\mathrm{CaP}$.

To characterize a role for $R$ heb in prostate tumorigenesis in vivo, we generated transgenic mice lines overexpressing $R$ heb in a prostate-specific manner. We engineered a transgenic construct in which a HA-tagged human cDNA encoding $R$ heb was cloned downstream from the prostate-specific small rat composite probasin promoter $\mathrm{ARR}_{2} \mathrm{~PB}$ (referred to as PB) (Zhang et al. 2000) and upstream of the SV40 polyA sequence (Fig. 1D, top panel). Pronuclear injection of the PB-HA-Rheb-SV40 polyA (PB-Rheb) construct resulted in the generation of seven founder mice as identified by genomic Southern blot analysis (Fig. 1D, bottom panel) and PCR (Supplemental Fig. 1A). Founder lines were expanded and prostate-specific expression of the transgene was identified in two different transgenic lines (47 and 50) by quantitative RT-PCR (Fig. 1E) and immunoprecipitation followed by Western blot analysis (Supplemental Fig. 1B). Human Rheb mRNA was detected in all the three lobes of the PB-Rheb transgenic mice (Fig. 1F). Importantly, no difference was observed in the expression of murine endogenous $R$ heb among our positive founders and wildtype controls (Supplemental Fig. 1C). Of note, the level of $R$ heb overexpression achieved in the prostate of the PB-Rheb transgenic mice was comparable with the level of Rheb overexpression observed in the human prostate cancer cell lines (Fig. 1G). To ensure that observed phenotypes were not a result of genomic insertion positional effects, we carried out all experiments with both transgenic lines with similar results.

To confirm the proper function of the PB-Rheb transgene, we analyzed the phosphorylation status of the S6 protein (Fig. 2A) and 4E-BP1 (Fig. 2B) as a readout of mTORC1 activation. Indeed, we observed elevated levels of phosphorylation for both proteins in the prostate of
A

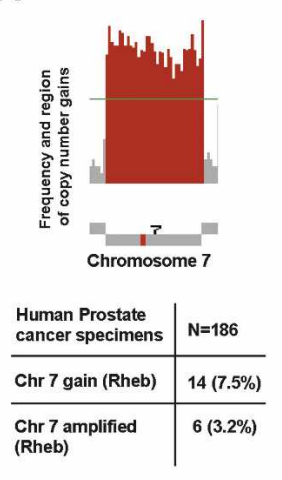

B
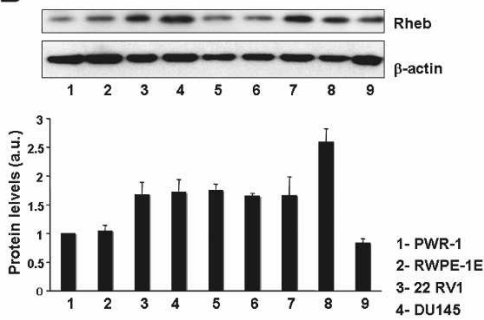

C
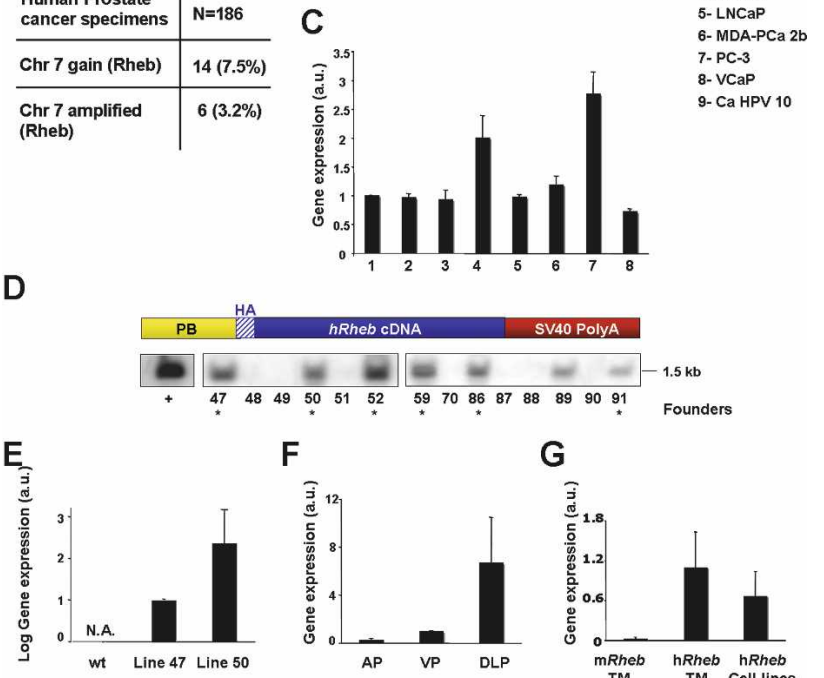

$F$

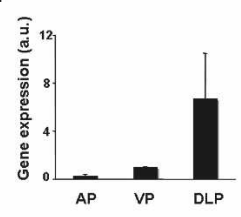

G

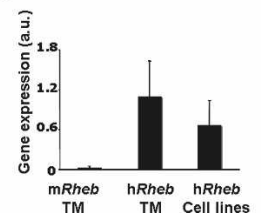

Figure 1. Overexpression of Rheb in human prostate cancer and generation of the PB-Rheb mouse model. (A, top panel) Schematic representation of copy number gain in chromosome 7 harboring $R h e b$. Red bars represent the frequency of regions gained on chromosome 7. (Bottom panel) Frequency of human prostate cancer specimens that show single copy number gain and amplification of chromosome 7. (B) Western blot analysis of Rheb in a panel of human prostate cell lines. Rheb levels are normalized to actin. (a.u.) Arbitrary units. Error bars show SD from three independent experiments. $(C)$ Quantitative RT-PCR analysis of the RNA from the same prostate cell lines (except for 9) showed in $B$ with a human Rheb-specific probe. Error bars show SD from three independent experiments. (D, top panel) Schematic representation of the transgenic construct. (Bottom panel) Southern blot analysis of genomic DNA from founder animals. As a control $(+)$ we used the vector harboring the transgenic construct digested as the genomic DNA from the tails. $(E)$ Quantitative RT-PCR analysis of the prostate mRNA from PB-Rheb mice with a human $R$ heb-specific probe. N.A. stands for not amplified. Error bars show SD from three independent experiments. (F) Differential expression of transgenic human Rheb in the three lobes of the prostate of wild-type and PB-Rheb mice analyzed by quantitative RT-PCR. (AP) Anterior prostate; (VP) ventral prostate; (DLP) dorsolateral prostate. Error bars show SD from three independent experiments. $(G)$ Level of endogenous murine $R h e b$ in the prostate of PB-Rheb transgenic mice (TM) and human $R h e b$ in the prostate cell lines showed in $C$, normalized to the level of human transgenic Rheb in TM analyzed by quantitative RT-PCR. Error bars show SD from three independent experiments.

PB-Rheb transgenic mice compared with their wild-type littermates. Overall, we generated mouse models of $R$ heb overexpression that can effectively potentiate mTORC1 activity in vivo in the prostatic epithelium.

Next, we examined the incidence of prostate-specific lesions and $\mathrm{CaP}$ in the $\mathrm{PB}-R$ heb mouse model. In cohorts of mice from the two PB-Rheb transgenic lines, we observed hyperplasia in the dorsolateral lobe of the prostate (DLP) as early 6 mo of age (line $47: 30 \%$ penetrance; line 50: $46 \%$ penetrance) (Fig. 2C,E). In these same two co- 
A

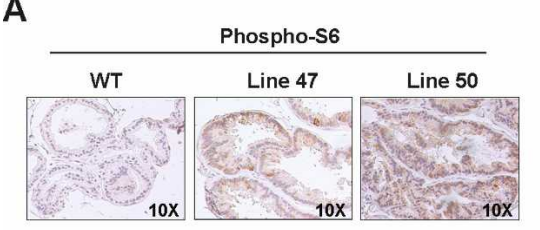

B

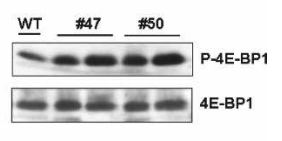

C

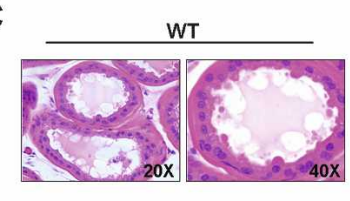

PB-Rheb
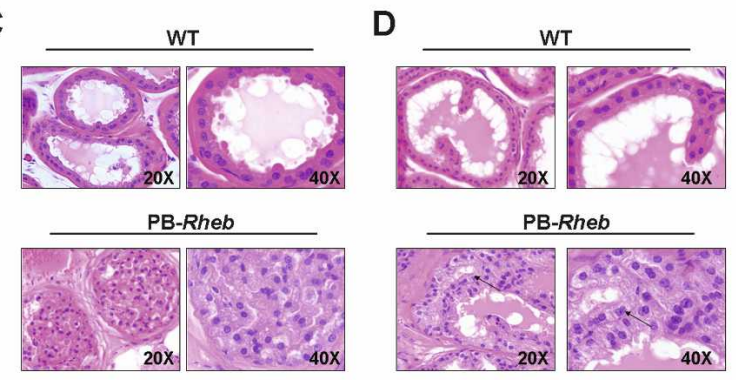

PB-Rheb

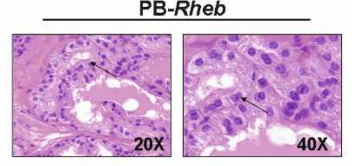

E

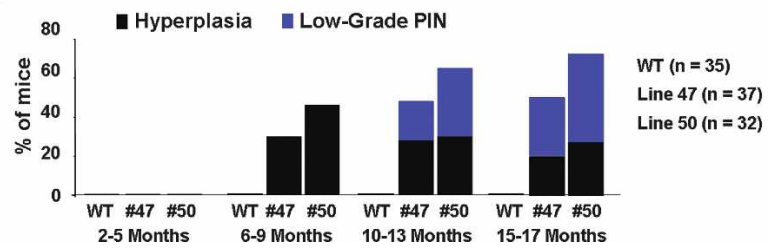

Figure 2. Analysis of the PB-Rheb mice prostates. $(A)$ IHC staining of prostate sections from wild-type and PB-Rheb mice with an antiphospho S6 antibody. $(B)$ Western blot analysis of protein lysates from wild-type and PB-Rheb mice. $(C) \mathrm{H} \& \mathrm{E}$ staining of DLP sections from 6-mo-old wild-type and PB-Rheb mice showing hyperplasia in the prostate of the transgenic mice. $(D) \mathrm{H} \& \mathrm{E}$ staining of DLP sections from 10-mo-old wild-type and $\mathrm{PB}-\mathrm{Rh} e b$ mice showing lowgrade PIN in the prostate of transgenic mice. An example of nuclear atypia is indicated by arrows. $(E)$ Cumulative incidence of hyperplasia and low-grade PIN over time in wild-type and PB-Rheb mice.

horts, transgenic mice developed low-grade prostatic intraepithelial neoplasia (LGPIN) lesions, characterized by prostatic glands containing multilayers of epithelial cells with features of nuclear atypia (line 47: 20\% penetrance; line $50: 35 \%$ penetrance) by 10 mo of age (Fig. 2D,E). At 17 mo of age (the oldest mice analyzed), the penetrance of LGPIN in the PB-Rheb transgenic mice was calculated to be $45 \%$ (line 50) and $32 \%$ (line 47) (Fig. 2E). This phenotype was also observed in the anterior prostate (AP) with lower penetrance. Conversely, we did not observe any morphological changes in ventral prostate (VP) at any age. Hyperplasia was confirmed by detection of the proliferation marker Ki-67, which was significantly increased in PB-Rheb mice relative to wild-type littermates (data discussed below; shown in Fig. 4F, below). These results are the first to demonstrate that $R h e b$ overexpression in vivo can drive aberrant growth of prostatic epithelia and the consequent development of prostatic hyperplasia and LGPIN.

The observation that PB-Rheb transgenic mice develop hyperplasia and LGPIN that does not progress to a higher stage of malignancy by 17 mo of age suggests that $R$ heb overexpression does not provide sufficient proliferative advantage per se to select for secondary oncogenic hits. We and others have demonstrated that the engagement of a senescence response is a potent tumor suppressor mechanism in vivo (for review, see Di Micco et al. 2007). Moreover, mTORC1 signaling has been implicated in this response via the ability of its downstream effector eIF4E to activate senescence (Ruggero et al. 2004). Therefore, we reasoned that a "brake" provided by senescence could explain the lack of more aggressive lesions in PB-Rheb transgenic mice. We addressed this question in vivo by measuring senescence in prostates of $\mathrm{PB}-$ Rhe $b$ transgenic mice. The analysis of cellular senescence by means of senescence-associated $\beta$-galactosidase (SA $\beta$-Gal) activity, a well-defined hallmark of senescence cells (Dimri et al. 1995), revealed that Rheb overexpression indeed triggers a senescence response in vivo (Fig. 3A). Importantly, we observed a differential response of the prostate lobes to Rheb overexpression; specifically there was a marked inverse relationship between the incidence of hyperplasia (Fig. 3B, left panel) and the level of senescence response in all three lobes of the mouse prostate (Fig. 3B, right panel). For instance, the DLP displayed the highest penetrance of hyperplasia in the absence of any senescence, whereas the VP showed no indications of hyperplasia with the highest levels of senescence among all three lobes. The AP displayed a medium level of senescence, which accordingly was accompanied by a mild hyperplastic phenotype. Moreover, the senescence response was confirmed in vitro upon infection of primary mouse embryonic fibroblasts (MEFs) with a HA-Rheb-PURO-IRES-GFP (HA$R$ heb-PIG)-encoding retrovirus (Supplemental Fig. 2). Interestingly, the senescence response induced by Rheb overexpression in vitro was abrogated by treatment with the mTORC1 inhibitor rapamycin (Supplemental Fig. 2). Together, these findings indicate that Rheb overexpres-

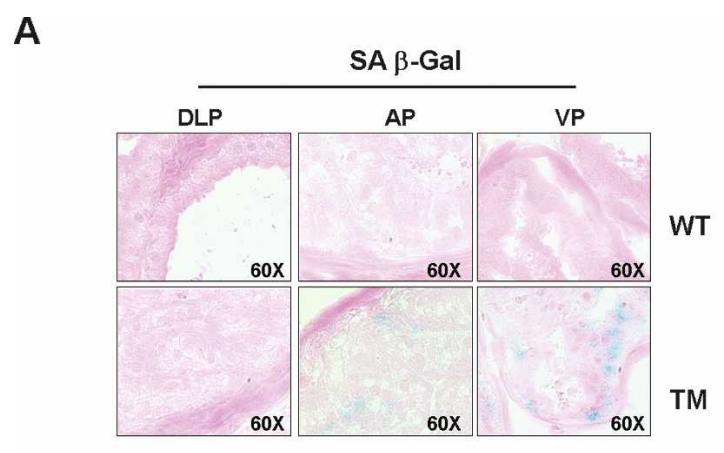

B

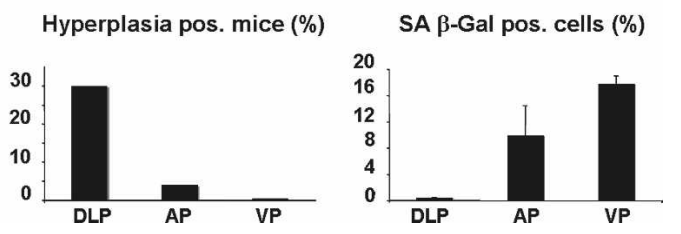

Figure 3. Inverse association between hyperplasia and cellular senescence in lobes of PB-Rheb mice prostates. $(A)$ Senescence-associated $\beta$-galactosidase staining (SA $\beta$-Gal) in the three different lobes of the prostate-DLP, AP, and VP-from 6- to 8-mo-old wild-type and PB-Rheb mice. ( $B$, left panel) Incidence of hyperplasia in the different lobes of the prostates from 6- to 8-mo-old PB-Rheb mice. (Right panel) Quantification of senescence in the different lobes of the prostates from 6- to 8-mo-old PB-Rheb mice measured by SA $\beta$-Gal staining. Quantifications were done on three representative sections from three mice. Pos. stands for positive. 
sion activates a clear and reproducible senescence response both in vitro and in vivo, a phenomenon that provides a likely explanation for the indolence of the lesions observed in the prostates of PB-Rheb transgenic mice.

Since $R$ heb overexpression per se does not produce full-blown $\mathrm{CaP}$, we explored the possibility that it could cooperate with additional oncogenic events for the development of more aggressive prostate malignancies. As aforementioned, loss of PTEN is a very common event in human CaP. We, therefore, analyzed the potential of synergism between Pten haploinsufficiency and Rheb overexpression for the development of $\mathrm{CaP}$. To do so, we intercrossed PB-Rheb transgenic mice with Pten heterozygous $\left(\right.$ Pten $\left.^{+/}\right)$mice, which develop high-grade PIN (HGPIN) with $\sim 50 \%$ penetrance by 9 mo of age (Di Cristofano et al. 2001). Strikingly, 10-mo-old PB-Rheb; Pten $^{+/-}$mice developed HGPIN with full penetrance in AP, compared with the incomplete $(40 \%)$ penetrance observed in Pten $^{+/-}$mice (Fig. 4A). Moreover, the individual HGPIN lesions in PB-Rheb;Pten ${ }^{+/-}$were markedly larger and more dysplastic compared with the lesions in Pten $^{+/-}$mice (Fig. 4B). Immunohistochemical (IHC) staining showed that Pten expression is similar between the two genotypes indicating that $R$ heb overexpression does not select for nor cause complete genetic loss of Pten (Supplemental Fig. 3A, left panel). We also assessed the consequences of Rheb overexpression and Pten haploinsufficiency on the activation status of the AktmTOR pathway. Akt activation, as measured by phosphorylated Akt, was comparatively elevated in HGPIN lesions from both PB-Rheb;Pten ${ }^{+/}$and Pten ${ }^{+-}$(Supplemental Fig. 3A, right panel). More importantly, we examined the Akt-mTOR pathway in preneoplastic prostate tissue from mice at the age of 5 mo. Firstly, we observed that S6 was more abundantly phosphorylated in preneoplastic prostatic tissue from PB-Rheb;Pten ${ }^{+/}$ compound mutants compared with $\mathrm{Pten}^{+/-}$mice (Fig. 4C). Furthermore, Akt phosphorylation was reduced by $R$ heb overexpression in vivo compared with wild-type controls (Fig. 4D), in association with higher S6K phosphorylation (Supplemental Fig. 3B). This finding is consistent with the activation of the negative feedback loop in which mTORC1 activation can inhibit the PI3K pathway by S6K1-mediated IRS-1 inhibition 1 (for review, see Guertin and Sabatini 2007). In contrast, in the context of Pten haploinsufficiency, overexpression of Rheb was inconsequential with respect to Akt phosphorylation status (Fig. 4D). Interestingly, and in full agreement with these findings, treatment of $\mathrm{PB}-R h e b$ transgenic mice with the rapamycin analog RAD001 relieved the negative feedback on Akt (Fig. 4E). Taken together, these findings are reminiscent of the inhibition of Akt signaling observed in Tsc2 heterozygosity, which is restored in the context of Pten haploinsufficiency (Ma et al. 2005; Manning et al. 2005).

To investigate the biological outcomes of Rheb overexpression per se, or in the context of Pten haploinsufficiency, we measured proliferation and senescence in preneoplastic prostate glands in PB-Rheb and PB-Rheb; Pten $^{+/-}$compound mutant mice. The expression of the proliferation marker Ki-67 was significantly increased $(P=0.0072)$ in PB-Rheb, confirming that $R$ heb overexpression induces a hyperplastic phenotype (Fig. 4F), in addition to cellular senescence (Fig. 3). Strikingly, Ki-67 staining in the prostates from PB-Rheb; ten $^{+}-$showed
A

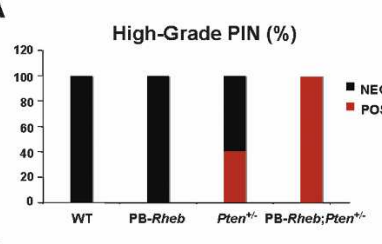

C

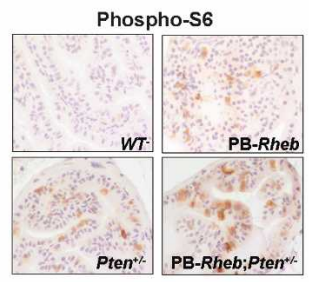

$\mathbf{F}$

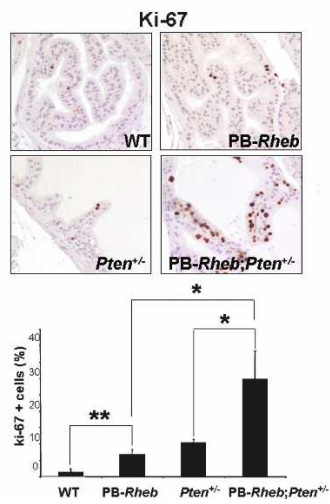

B

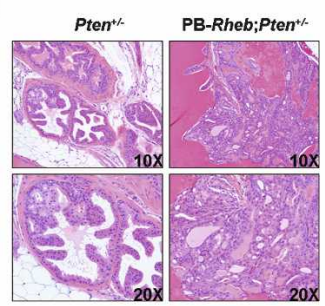

D

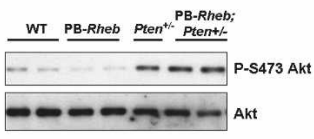

E

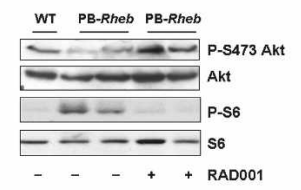

G

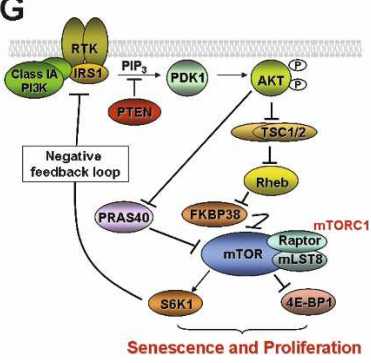

Figure 4. Cooperativity of Rheb overexpresison and Pten haploinsufficiency. $(A)$ Incidence of HGPIN in the AP from five 10-mo-old mice of each genotype. $(B)$ Histopathological $(\mathrm{H} \& E)$ analysis of the AP sections from 10-mo-old mice of each genotype. (C) IHC staining of prostate sections from 5-mo-old mice of each genotype with an anti-phospho $\mathrm{S} 6$ antibody. $(D)$ Western blot analysis of protein extracts from AP of 5-mo-old mice of the indicated genotype. $(E)$ Western blot analysis of protein extracts from mice of the indicated genotype treated or not with RAD001. $(F)$ Ki-67 staining on prostate sections from 6-mo-old mice of the indicated genotypes. Bottom panel: quantification of $\mathrm{Ki}-67$. A representative count in triplicate out of three with similar results is shown with standard deviations. (G) Molecular model of mTORC1 activation by Rheb leading to senescence and proliferation and feedback inhibition of the PI3K pathway.

that Rheb overexpression synergizes with Pten haploinsufficiency to markedly enhance proliferation (Fig. 4F). Conversely, we observed that the proportion of glandular senescent cells in prostate of PB-Rheb; Pten $^{+/}$was only mildly increased when compared with $\mathrm{PB}-R$ heb mice (Supplemental Fig. 3C). Overall, Rheb overexpression in the context of Pten haploinsufficiency leads to a marked increase in proliferation in one pool of cells, with a concomitant and slight enhancement of cellular senescence response in a distinct pool of cells. Thus, activation of mTORC1 by Rheb overexpression can direct a cell toward two fates: proliferation or senescence. The mechanisms dictating the choice of whether a cell will undergo the proliferative or senescence response are currently unknown, and this decision may be of stochastic nature. In our study, we revealed that Pten haploinsufficiency can shift the balance of these two events toward proliferation and consequently promote a more severe phenotype.

Our results are consistent with a model whereby the 
engagement of a senescence response, in addition to activation of a negative feedback loop that inhibits the $\mathrm{PI} 3 \mathrm{~K} / \mathrm{AKT}$ pathway, contributes to the failure of $R$ heb overexpression alone to induce a full-blown in situ cancerous lesion (model in Fig. 4G). We propose that the oncogenic cooperativity between $R$ heb overexpression and heterozygous Pten loss is based on (1) direct potentiation of mTOR activity by Rheb overexpression, in conjunction with (2) relief of Akt inhibition conferred by the Pten haploinsufficiency-mediated "breaking" of the negative feedback loop (model in Fig. 4G). We postulate that the reason why partial Pten loss potentiates differentially proliferation rather than senescence is that the threshold of pathway activation needed to trigger a fullblown senescence response is not yet reached by combined Pten heterozygous loss and Rheb overexpression: a scenario similar to what is observed in Pten hypomorphic conditions (Trotman et al. 2003). Relief of Akt inhibition by Pten haploinsufficiency, on one hand, increases the level of mTORC1 activation achieved by $R$ heb overexpression alone through regulation of two different components of the pathway: (1) phosphorylation and inhibition of PRAS40, which has been shown to compete with Rheb on mTORC1 activity (for review, see Guertin and Sabatini 2007), and (2) TSC2 phopshorylation, which results in a higher amount of GTP-bound Rheb and thereby enhanced activity of exogenous Rheb. On the other hand, Akt activation is also known to drive additional signals through activation of its downstream effectors that promote cell survival and growth (Manning and Cantley 2007), thereby further contributing to tumorigenicity.

We speculate that, during the evolution of $\mathrm{CaP}$, cells with overactive PI3K/AKT signaling may select for $R$ heb overexpression as a mechanism to reinforce a proliferative response. We showed previously that complete loss of Pten is invariably associated with a p53-dependent cellular senescence response (Chen et al. 2005). Thus, in a PTEN heterozygous state and during the initiation of $\mathrm{CaP}$, Rheb overexpression may be selected because it markedly enhances proliferation, while complete PTEN loss is only compatible with circumstances where the cellular senescence response is fully evaded through loss of $\mathrm{p} 53$, which in $\mathrm{CaP}$ is only observed in advanced metastatic lesions (Navone et al. 1999; Qian et al. 2002).

Finally, because Rheb is potentially "druggable" in view of its specific enzymatic activity, our findings suggest that Rheb inhibition could prove useful in tumors displaying aberrant Rheb activity, hence providing the basis for novel therapeutic strategies for the prevention of prostate cancer progression toward invasion and metastasis.

\section{Materials and methods}

Cell culture and protein analysis

The human prostate cancer cell lines PWR-1E, RWPE-1, 22 RV1, DU145, LNCaP, MDA-PCa 2b, PC-3, VCaP, Ca HPV 10 (American Type Culture Collection) were cultured according to the manufacturer instructions. Cell lysates were prepared with RIPA buffer (1× PBS, 1\% Nonidet P40, $0.5 \%$ sodium deoxycholate, $0.1 \%$ SDS, protease inhibitor cocktail [Roche]) and cleaned by centrifugation. The following antibodies were used for Western blot analysis: rabbit polyclonal anti-Rheb (IN; ProSci), mouse anti- $\beta$-actin (AC-72; Sigma), anti-phospho p70 S6 Kinase (Thr389) (Cell Signaling Technology), anti-p70 S6 Kinase (Cell Signaling Technology), anti-phospho 4E-BP1 (Ser65) (Cell Signaling Technology), anti-4EBP1 (Cell Signaling Technology), anti-HA (Covance), anti-phospho-Akt (S473) (Cell Signaling Technology), anti-Akt (Cell Signaling Technology).
Generation of transgenic mice and Southern blot analysis Human Rheb cDNA (UMR cDNA Resource Center, University of Missouri-Rolla) was HA-tagged and transferred to the EcoRI-digested vector pSV containing the probasin promoter sequence (a kind gift from Dr. Pradip Roy-Burman). The fragment containing PB-HA-Rheb-SV40 poly(A) was released by digestion with NotI and KpnI and injected into the pronuclei of fertilized oocytes in a B6/CBA F1 hybrid background. The Southern blot analysis was performed as reported previously (Song et al. 2002).

\section{Quantitative RT-PCR}

Total RNA was prepared from mice prostates using the Trizol method (Invitrogen) and from the cell lines, described above, using the RNeasy Mini Kit (Qiagen). cDNA was obtained with Transcriptor (Roche). TaqMan probes were obtained from Applied Biosystems. Amplifications were run in a 7900 Real-Time PCR System (Applied Biosystems). Each value was adjusted by using Glucuronidase B levels as a reference.

\section{Histopathology and immunohistochemistry}

Mice were autopsied, prostates were extracted and fixed in $10 \%$ neutralbuffered formalin (Sigma) overnight, subsequently washed once with PBS, transferred into 50\% ethanol, and stored in $70 \%$ ethanol. Prostate lobes were embedded in paraffin, sectioned, and stained with hematoxylin and eosin $(H \& E)$ in accordance with standard procedures. Sections were stained with the following antibodies: anti-phospho-S6 (S235/S236) (Cell Signaling Technology); PTEN (Ab-5, NeoMarkers); anti-phosphoAkt (S473) (Cell Signaling Technology); Ki-67 (Novacastra).

\section{Senescence detection in vivo}

Prostates were frozen in OTC, and frozen sections $5 \mu \mathrm{m}$ thick were stained for $\beta$-galactosidase activity using the Senescence Detection Kit (Calbiochem). Quantifications were performed on three representative sections from three mice for each genotype.

\section{PB-Rheb;Pten ${ }^{+/-}$mice generation}

PB-Rheb transgenic mice were crossed with Pten $^{+/-}$mice (Di Cristofano et al. 1998) through two generations of breeding to ensure normalization of the background strains. Pten genotyping by PCR on tail DNA was performed as described previously (Di Cristofano et al. 1998).

Treatment of mice with RAD001

RAD001 (everolimus) was obtained from Novartis and was administered by oral gavage daily at $10 \mathrm{mg} / \mathrm{kg}$ a day for $1 \mathrm{wk}$.

\section{Acknowledgments}

We thank R. Bernardi, J.G. Clohessy, and L. Poliseno for critical discussions and help. We thank Novartis for RAD001. C.N. was supported in part by a fellowship from the American-Italian Cancer Foundation. L.S. is supported by an HFSP Long-Term Fellowship, and A.C. is supported by an EMBO long-term fellowship. This work is supported through MMHCC and NCI grants.

\section{References}

Bai, X., Ma, D., Liu, A., Shen, X., Wang, Q.J., Liu, Y., and Jiang, Y. 2007. Rheb activates mTOR by antagonizing its endogenous inhibitor, FKBP38. Science 318: 977-980.

Chen, Z., Trotman, L.C., Shaffer, D., Lin, H.K., Dotan, Z.A., Niki, M., Koutcher, J.A., Scher, H.I., Ludwig, T., Gerald, W., et al. 2005. Crucial role of p53-dependent cellular senescence in suppression of Ptendeficient tumorigenesis. Nature 436: 725-730.

Di Cristofano, A., Pesce, B., Cordon-Cardo, C., and Pandolfi, P.P. 1998. Pten is essential for embryonic development and tumour suppression. Nat. Genet. 19: 348-355.

Di Cristofano, A., De Acetis, M., Koff, A., Cordon-Cardo, C., and Pandolfi, P.P. 2001. Pten and p27 ${ }^{\mathrm{KIP} 1}$ cooperate in prostate cancer tumor suppression in the mouse. Nat. Genet. 27: 222-224.

Di Micco, R., Fumagalli, M., and di Fagagna, F. 2007. Breaking news: High-speed race ends in arrest-How oncogenes induce senescence. Trends Cell Biol. 17: 529-536.

Dimri, G.P., Lee, X., Basile, G., Acosta, M., Scott, G., Roskelley, C., 
Medrano, E.E., Linskens, M., Rubelj, I., Pereira-Smith, O., et al. 1995. A biomarker that identifies senescent human cells in culture and in aging skin in vivo. Proc. Natl. Acad. Sci. 92: 9363-9367.

Easton, J.B. and Houghton, P.J. 2006. mTOR and cancer therapy. Oncogene 25: 6436-6446.

Gao, X., Zhang, Y., Arrazola, P., Hino, O., Kobayashi, T., Yeung, R.S., Ru, B., and Pan, D. 2002. Tsc tumour suppressor proteins antagonize amino-acid-TOR signalling. Nat. Cell Biol. 4: 699-704.

Garami, A., Zwartkruis, F.J., Nobukuni, T., Joaquin, M., Roccio, M., Stocker, H., Kozma, S.C., Hafen, E., Bos, J.L., and Thomas, G. 2003. Insulin activation of Rheb, a mediator of mTOR/S6K/4E-BP signaling, is inhibited by TSC1 and 2. Mol. Cell 11: 1457-1466.

Gray, I.C., Stewart, L.M., Phillips, S.M., Hamilton, J.A., Gray, N.E., Watson, G.J., Spurr, N.K., and Snary, D. 1998. Mutation and expression analysis of the putative prostate tumour-suppressor gene PTEN. Br. J. Cancer 78: 1296-1300.

Guertin, D.A. and Sabatini, D.M. 2007. Defining the role of mTOR in cancer. Cancer Cell 12: 9-22.

Inoki, K., Li, Y., Xu, T., and Guan, K.L. 2003. Rheb GTPase is a direct target of TSC2 GAP activity and regulates mTOR signaling. Genes \& Dev. 17: 1829-1834.

Li, Y., Corradetti, M.N., Inoki, K., and Guan, K.L. 2004. TSC2: Filling the GAP in the mTOR signaling pathway. Trends Biochem. Sci. 29: 3238.

Ma, L., Teruya-Feldstein, J., Behrendt, N., Chen, Z., Noda, T., Hino, O., Cordon-Cardo, C., and Pandolfi, P.P. 2005. Genetic analysis of Pten and Tsc2 functional interactions in the mouse reveals asymmetrical haploinsufficiency in tumor suppression. Genes \& Dev. 19: 17791786.

Majumder, P.K. and Sellers, W.R. 2005. Akt-regulated pathways in prostate cancer. Oncogene 24: 7465-7474.

Manning, B.D. and Cantley, L.C. 2003. Rheb fills a GAP between TSC and TOR. Trends Biochem. Sci. 28: 573-576.

Manning, B.D. and Cantley, L.C. 2007. AKT/PKB signaling: Navigating downstream. Cell 129: 1261-1274.

Manning, B.D., Tee, A.R., Logsdon, M.N., Blenis, J., and Cantley, L.C. 2002. Identification of the tuberous sclerosis complex-2 tumor suppressor gene product tuberin as a target of the phosphoinositide 3-kinase/akt pathway. Mol. Cell 10: 151-162.

Manning, B.D., Logsdon, M.N., Lipovsky, A.I., Abbott, D., Kwiatkowski, D.J., and Cantley, L.C. 2005. Feedback inhibition of Akt signaling limits the growth of tumors lacking Tsc2. Genes \& Dev. 19: 17731778.

Navone, N.M., Labate, M.E., Troncoso, P., Pisters, L.L., Conti, C.J., von Eschenbach, A.C., and Logothetis, C.J. 1999. p53 mutations in prostate cancer bone metastases suggest that selected p 53 mutants in the primary site define foci with metastatic potential. J. Urol. 161: 304308.

Potter, C.J., Pedraza, L.G., Huang, H., and Xu, T. 2003. The tuberous sclerosis complex (TSC) pathway and mechanism of size control. Biochem. Soc. Trans. 31: 584-586.

Qian, J., Hirasawa, K., Bostwick, D.G., Bergstralh, E.J., Slezak, J.M. Anderl, K.L., Borell, T.J., Lieber, M.M., and Jenkins, R.B. 2002. Loss of p53 and c-myc overrepresentation in stage $\mathrm{T}^{2-3} \mathrm{~N}^{1-3} \mathrm{M}^{0}$ prostate cancer are potential markers for cancer progression. Mod. Pathol. 15: $35-44$.

Ruggero, D., Montanaro, L., Ma, L., Xu, W., Londei, P., Cordon-Cardo, C., and Pandolfi, P.P. 2004. The translation factor eIF-4E promotes tumor formation and cooperates with c-Myc in lymphomagenesis. Nat. Med. 10: 484-486.

Song, Z., Wu, X., Powell, W.C., Cardiff, R.D., Cohen, M.B., Tin, R.T., Matusik, R.J., Miller, G.J., and Roy-Burman, P. 2002. Fibroblast growth factor 8 isoform B overexpression in prostate epithelium: A new mouse model for prostatic intraepithelial neoplasia. Cancer Res. 62: $5096-5105$

Tapon, N., Ito, N., Dickson, B.J., Treisman, J.E., and Hariharan, I.K. 2001 The Drosophila tuberous sclerosis complex gene homologs restrict cell growth and cell proliferation. Cell 105: 345-355.

Tee, A.R., Anjum, R., and Blenis, J. 2003. Inactivation of the tuberous sclerosis complex-1 and -2 gene products occurs by phosphoinositide 3-kinase/Akt-dependent and -independent phosphorylation of tuberin. J. Biol. Chem. 278: 37288-37296.

Trotman, L.C., Niki, M., Dotan, Z.A., Koutcher, J.A., Di Cristofano, A.,
Xiao, A., Khoo, A.S., Roy-Burman, P., Greenberg, N.M., Van Dyke, T., et al. 2003. Pten dose dictates cancer progression in the prostate. PLOS Biol. 1: E59. doi: 10.1371/journal.pbio.0000059.

Whang, Y.E., Wu, X., Suzuki, H., Reiter, R.E., Tran, C., Vessella, R.L., Said, J.W., Isaacs, W.B., and Sawyers, C.L. 1998. Inactivation of the tumor suppressor PTEN/MMAC1 in advanced human prostate cancer through loss of expression. Proc. Natl. Acad. Sci. 95: 5246-5250.

Wullschleger, S., Loewith, R., and Hall, M.N. 2006. TOR signaling in growth and metabolism. Cell 124: 471-484.

Zhang, J., Gao, X., Thomas, T.Z., Kasper, S., and Matusik, R.J. 2000. A small composite probasin promoter confers high levels of prostatespecific gene expression though regulation by androgens and glucocoticoids in vitro and in vivo. Endocrinology 141: 4698-4710.

Zhang, Y., Gao, X., Saucedo, L.J., Ru, B., Edgar, B.A., and Pan, D. 2003. Rheb is a direct target of the tuberous sclerosis tumour suppressor proteins. Nat. Cell Biol. 5: 578-581. 


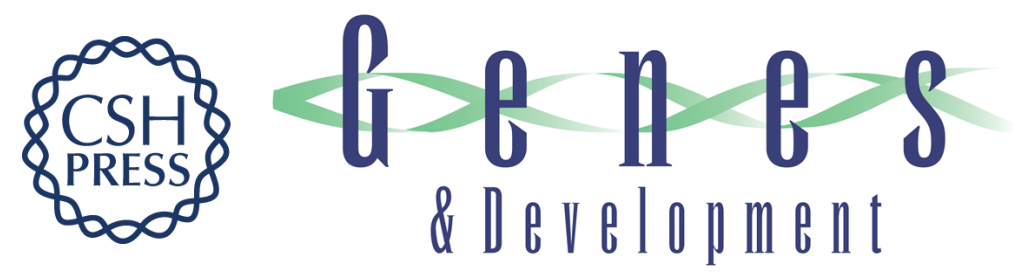

\section{Aberrant Rheb-mediated mTORC1 activation and Pten haploinsufficiency are cooperative oncogenic events}

Caterina Nardella, Zhenbang Chen, Leonardo Salmena, et al.

Genes Dev. 2008, 22:

Access the most recent version at doi:10.1101/gad.1699608

Supplemental http://genesdev.cshlp.org/content/suppl/2008/07/29/22.16.2172.DC1
Material

References This article cites 31 articles, 8 of which can be accessed free at:

http://genesdev.cshlp.org/content/22/16/2172.full.html\#ref-list-1

License

Email Alerting Receive free email alerts when new articles cite this article - sign up in the box at the top

Service right corner of the article or click here.

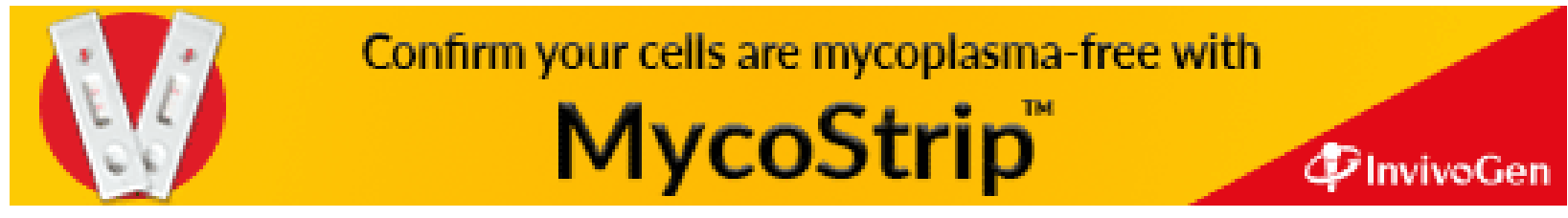

